\title{
Usefulness of cardio-magnetic resonance in the diagnosis and evaluation of pulmonary hypertension
}

\author{
Pilar Egea-Serrano ${ }^{1 *}$, Jeremy Collinst ${ }^{2}$, Maria M Izquierdo ${ }^{3}$, Timothy Scanlon ${ }^{4}$, Philip Hodnett $^{4}$, Eduardo Segoviat ${ }^{1}$, \\ Manuel Sancho ${ }^{5}$, James Carr ${ }^{4}$
}

From 2011 SCMR/Euro CMR Joint Scientific Sessions

Nice, France. 3-6 February 2011

\section{Introduction}

The diagnosis of PH should be based on clinical suspicious and specific complementary tests. CMR has no acoustic window limitation, it is the gold standard for quantification of right ventricular (RV) function, it is the main test for congenital heart disease (CHD) and finally it gives more comprehensive information.

\section{Purpose}

To show how useful the CMR was for patients referred for heart failure (HF), regarding the identification and severity of pulmonary hypertension (PH) not previously assessed by other non-invasive techniques.

\section{Methods}

We reviewed patients referred to our institution from January 2008 to March 2010 for further evaluation of HF who were firstly diagnosed of PH by CMR. The study was performed on a $1.5 \mathrm{~T}$ Avanto scanner and included: cine images, phase-contrast sequence, timeresolved angiography for aorta and pulmonary artery, and inversion recovery sequence. The data analyzed was: ventricular volumes and functions, flows, interventricular septum (IVS) motion, and assessment of late gadolinium enhancement (LGE).

\section{Results}

20 patients were included. Eleven (55\%) were female and the mean age was 47 . Following the Dana Point classification 2008: 10 belonged to group 1; 4 to group 2; 1 to group 3; 1 to group 4; and 4 patients had mixed conditions. All patients presented high RV volumes (end-dyastolic $209 \pm$ $88 \mathrm{~mL}$; and end-systolic $128 \pm 81,7 \mathrm{~mL}$ ). The RV ejection

${ }^{1}$ Cardiology Department. Hospital de Cabueñes, Gijón, Spain

Full list of author information is available at the end of the article fraction (RVEF) was $41 \pm 16 \%$. The RV outflow tract was enlarged $(30 \pm 5 \mathrm{~mm})$ and pulmonary artery (PA) too (36 $\pm 7,5 \mathrm{~mm})$. RV wall thickness was $4,2 \pm 1,4 \mathrm{~mm}$. Pulmonary peak velocity was $105,7 \pm 76,8 \mathrm{mmHg}$, with moderate regurgitation. IVS motion classified as: $50 \%$ flattening, $35 \% \mathrm{D}$-shaped, normal only in 1 . The left ventricular (LV) overall function was related to the baseline disease. The systolic RV/LV volume ratio was $1,9 \pm 0,8$ and the diastolic one was $1,6 \pm 0,6$. LGE was present in $60 \%$, mostly in basal RV and LV insertion points; additionally in LV inferior wall in one patient suggesting myocardial infarct, and another patient presented transmural LGE in inferior apical wall. CMR angiography was of value as it allowed orthogonal measures of PA and showed features of chronic thromboembolic disease, pulmonary vein stenosis and discovered CHD.

\section{Conclusions}

CMR is able not only to diagnose $\mathrm{PH}$ but also provide its severity and etiology, offering prognosis and guiding management / follow-up within a single examination.

\section{Author details \\ ${ }^{1}$ Cardiology Department. Hospital de Cabueñes, Gijón, Spain. ${ }^{2}$ Cardiovascular Imaging. Radiology Department. Northwestern Memorial Hospital., Chicago, IL, USA. ${ }^{3}$ Cardiology Department. Northwestern Memorial Hospital., Chicago, IL, USA. ${ }^{4}(1)$ Cardiovascular Imaging. Radiology Department. Northwestern Memorial Hospital, Chicago, IL, USA. ${ }^{5}$ Cardiology Department. Hosital U \\ Puerta del Mar, Cádiz, Spain.}

Published: 2 February 2011

doi:10.1186/1532-429X-13-S1-P335

Cite this article as: Egea-Serrano et al:: Usefulness of cardio-magnetic resonance in the diagnosis and evaluation of pulmonary hypertension. Journal of Cardiovascular Magnetic Resonance 2011 13(Suppl 1):P335.

\section{C)

๑ 2011 Egea-Serrano et al; licensee BioMed Central Ltd. This is an open access article distributed under the terms of the Creative Commons Attribution License (http://creativecommons.org/licenses/by/2.0), which permits unrestricted use, distribution, and reproduction in any medium, provided the original work is properly cited. 